\title{
ONE-STEP DISTORTION SIMULATION OF PULSED LASER WELDING WITH MULTI-PHYSICS INFORMATION
}

\author{
Kim, S.; Lee, W. \& Kim, D. \\ Department of Mechanical Engineering, Sogang University, 1 Shinsoo-dong, Mapo-go, Seoul, \\ Republic of Korea \\ E-Mail: dckim@sogang.ac.kr
}

\begin{abstract}
The pulsed laser welding technique involves complex physical mechanisms, which demand a multiphysics welding simulation for reliable computational analysis. Although the multi-physics simulation provides high accuracy in detailed welding information, it is difficult to apply in practice, as it requires vast computational resources for systematic analysis. We propose a highly efficient and reliable simulation technique based on multi-physics analysis and mechanical analysis. The developed technique is used to efficiently and reliably simulate a complete model of a nuclear fuel spacer grid by introducing a virtual welding distortion that exports the thermo-mechanical information. This study has the potential to develop the computational analysis and optimization of a sequence of pulse laser welding in a nuclear fuel spacer grid.

(Received in March 2014, accepted in October 2014. This paper was with the authors 1 month for 2 revisions.)
\end{abstract}

Key Words: Pulsed Laser Welding, Multi-Physics Analysis, Welding Distortion, Nuclear Fuel Spacer Grid, Finite Element Analysis

\section{INTRODUCTION}

Conventional welding methods, such as arc welding, are widely used for joining metal specimens with a high-strength bond. However, the accumulated thermal energy and temperature gradients result in large welding distortions and residual stress in the specimens. To prevent this side-effect of the welding assembly process, several welding techniques have been proposed, such as laser spot, friction stir, and magnetic pulse welding. Laser spot welding is appropriate for minimizing the thermal distortion and residual stress. A laser heat source focuses a large amount of energy in a small area, and the pulsed welding requires less energy than continuous welding in joining components. Thus, pulsed welding generates a narrow heat-affected zone (HAZ) that is closely related to the welding distortion. Pulsed laser welding consists of several overlapping welding spots that are repeatedly heated rapidly and cooled down over a period of seconds.

Each spot welding technique involves complex physical mechanisms, such as keyholes. These physical mechanisms are closely related to the parameters of pulse welding, such as the peak power, duration, and frequency of the pulses. Thus, there have been many attempts to parameterize the laser spot welding technique and to analyse the effect of each parameter on the welding distortion. Ghosh predicted the dimensions of the HAZ arising from submerged arc welding and optimized the input parameters, such as power and speed, in order to get better bead quality and minimum HAZ width [1]. Bukvic studied the fatigue failure at the HAZ of a bi-material welded joint [2]. Weckman et al. analysed the relationships between the parameters of laser spot welding, such as the pulse duration and power density, and different diameters and depths of the welding spot [3]. Tzeng investigated the effects of the welding parameters, including the mean power, pulse energy, and the traverse speed, on the dimensions of the welding spot and the weldability [4]. Fuerschbach and Eisler predicted the size of a welding spot from the experimentally measured pulse energy and duration [5]. Tadamalle studied the influence of the transverse speed and peak power on the geometry of 
the weld bead, duty cycle, pulse overlap, and energy density [6]. Because pulsed laser welding generates a small volume of molten material, it has been employed to reduce changes in the material properties of the welded part. Vegelj applied pulsed laser welding with adaptive power to weld electrical laminations, and minimized the magnetic loss of the stator or rotor stack in the electromotor [7].

Computational analysis has recently become a more reliable approach for the study and design of the welding process. It can predict not only mechanical behaviours such as the welding distortion, residual stress, and strain, but also thermo-physical conditions including the temperature field, heat flux, and phase transition. To obtain verified simulation results that may replace pulsed laser welding experiments, the computational study must consider the complex physical mechanisms, including rapid changes in temperature, phase transitions induced by the laser heat source, welding distortion, and the generation of residual stress, that occur during the welding process.

Rosenthal derived the first analytic solution, which forms the foundation of early models of laser welding [8]. Mazumder and Steen constructed the first model with a finite difference method that simulated a continuous laser welding process with a kinetic Gaussian heat source [9]. Frewin and Scott developed a model with the finite element method to calculate the temperature field induced by pulsed laser welding [10]. He et al. developed a transient heat transfer and fluid flow model based on the solution of mass, momentum, and energy conservation in the weld pool to predict the weld pool geometry, weld thermal cycles, and various solidification parameters [11]. Ehlen et al. investigated the shape of a melt pool during laser spot welding using an axisymmetric two-dimensional control volume method [12]. Moraitis and Labeas performed a numerical investigation of an aluminium lap joint with laser welding to study mechanical behaviours such as residual stress and welding distortion [13].

Recently, we performed a multi-physics simulation that described pulsed laser welding in thin sheets of Zircaloy-4, and compared the dimensions of the weld to experimental results with great accuracy [14]. Ferro and Tiziani verified the characterizations of electron beam welding obtained by multi-physics simulations with experimental data [15]. Joshi et al. characterized the parameters of a welding heat source in two overlapping beads with multiphysics simulations [16].

The multi-physics welding simulation successfully describes the behaviour of the welding species, such as the dimensions of the weld spot, including the HAZ and melting zone, and the thermo-mechanical conditions, including residual stress and welding distortion. Nevertheless, there are some limits in applying multi-physics analysis to practical welding applications. Firstly, as a multi-physics analysis considers almost every complex welding mechanism, it consumes vast computing resources. Thus, it is applicable only to a limited model with dimensions in the order of millimetres to several centimetres, which is insufficient for a general welding study or design of a welding process. Secondly, the multi-physics simulation requires long calculation times with poor efficiency, even for small species. Thus, especially for the study and design of a welding assembly process for typical complex systems, a reliable and efficient simulation approach that can overcome the limits of multiphysics simulation is necessary.

In this paper, we propose a highly reliable and efficient simulation technique for the pulsed laser welding of a complicated system: a nuclear fuel spacer grid. From the computational analysis of a multi-physics simulation, we developed an efficient one-step simulation process by coupling a multi-physics analysis with a mechanical distortion analysis. By introducing a virtual welding distortion that exports the resultant thermo-mechanical information in the welding region, the distortion generated in the entire model by each welding process can be efficiently simulated, even in a complete model of the nuclear fuel 
spacer grid. The developed welding simulation technique provides reliable accuracy and significant efficiency compared to the basic multi-physics simulation.

\section{MODELLING}

\subsection{Welding assembly process for a nuclear spacer grid}

There are various designs for a nuclear fuel spacer grid. Here, we consider a nuclear spacer grid assembled with 26 inner strips and four outer strips enclosing the inner strips. As shown in Fig. 1, it is composed of a $14 \times 14$ orthogonal array for nuclear fuel rods. The inner strip has projected parts supporting the nuclear fuel rods. The top and bottom intersections between the inner strips are assembled by laser spot welding. Joints between the inner strips and outer strips are assembled by seam welding with a pulsed laser, with dimensions of $2.5 \mathrm{~mm}$ in width and $12.5 \mathrm{~mm}$ in length. The thickness of an inner strip is $0.5 \mathrm{~mm}$ and the distance between the strips is $14 \mathrm{~mm}$. As with the dimensions of the repeated orthogonal unit formed with inner strips, the specifications of the welding procedure are uniform.

In order to develop the proposed technology, pulsed seam welding that induces severe welding distortion is considered. An accurate computation for pulsed laser welding incorporating multiple mechanisms demands a highly detailed model with a large number of elements. A laser heat source with high thermal energy density is introduced in a very short span of time, which induces a rapid change in temperature at each welding spot. In numerical calculations, such a drastic gradient of conditions over a narrow area generally causes numerical instability. Thus, to avoid the instability, a delicately refined mesh needs to be generated in the welding region. However, a sufficiently accurate model of a $14 \times 14$ orthogonal array for nuclear fuel rods leads to extremely low computational efficiency. Thus, for efficient computational analysis, simplified models are constructed with a few repeated units of the nuclear fuel spacer grid, as shown in Fig. 2. One model is composed of four repeating orthogonal units, with one line of intersection between the inner strip and the outer strip with pulsed laser welding. The other model has $4 \times 4$ orthogonal units and three welded regions on each outer strip. In a welding region, a carefully refined mesh is generated with more than $80 \%$ of the total number of elements for accurate computation of pulsed laser welding. As the elements become smaller and denser, the intense stress that causes instability can be dispersed. The simplified models, which are $2 \times 2$ and $4 \times 4$, have only two welding regions and 58,373 and 69,661 elements with 74,748 and 98,532 nodes, respectively. Most of the elements are densely packed in the welding region. The sizes of the $2 \times 2$ and $4 \times 4$ models are $30 \mathrm{~mm} \times 30 \mathrm{~mm} \times 27 \mathrm{~mm}$ and $60 \mathrm{~mm} \times 60 \mathrm{~mm} \times 27 \mathrm{~mm}$, respectively. In the $z$-direction, half of the nuclear fuel spacer grid is modelled, as the grid is symmetric except at the deflected edges. The described dimensions, including the protrusions in the inner strip and gaps in the intersection region, are obtained from measurements. The material of the grid is Zircaloy-4, which is mainly composed of zirconium and tin. The material properties of Zircaloy-4 are obtained from references.

Table I: Chemical composition and properties of Zircaloy-4.

\begin{tabular}{|c|c|l|c|}
\hline \multicolumn{2}{|c|}{ Chemical composition (\%) } & \multicolumn{2}{|c|}{ Material properties } \\
\hline $\mathrm{Cr}$ & 0.1 & Thermal conductivity (W/mK) & $13.5 \sim 55$ \\
\hline $\mathrm{Fe}$ & 0.2 & Specific Heat (J/kgK) & $30 \sim 90$ \\
\hline $\mathrm{O}$ & 0.12 & Density $(\mathrm{g} / \mathrm{cc})$ & 6.5 \\
\hline $\mathrm{Sn}$ & 1.4 & Elastic modulus $(\mathrm{GPa})$ & $110 \sim 10$ \\
\hline $\mathrm{Zr}$ & Balance & Yield strength $(\mathrm{MPa})$ & $400 \sim 80$ \\
\hline
\end{tabular}


Siefken et al. studied the thermal properties of Zircaloy-4, including the density and thermal strain with respect to temperature [17]. Han et al. obtained the yield strength and elastic modulus of Zircaloy-4 as a function of temperature by conducting the Gleeble test [14]. Table I shows the chemical composition and material properties of Zircaloy-4 that we considered.

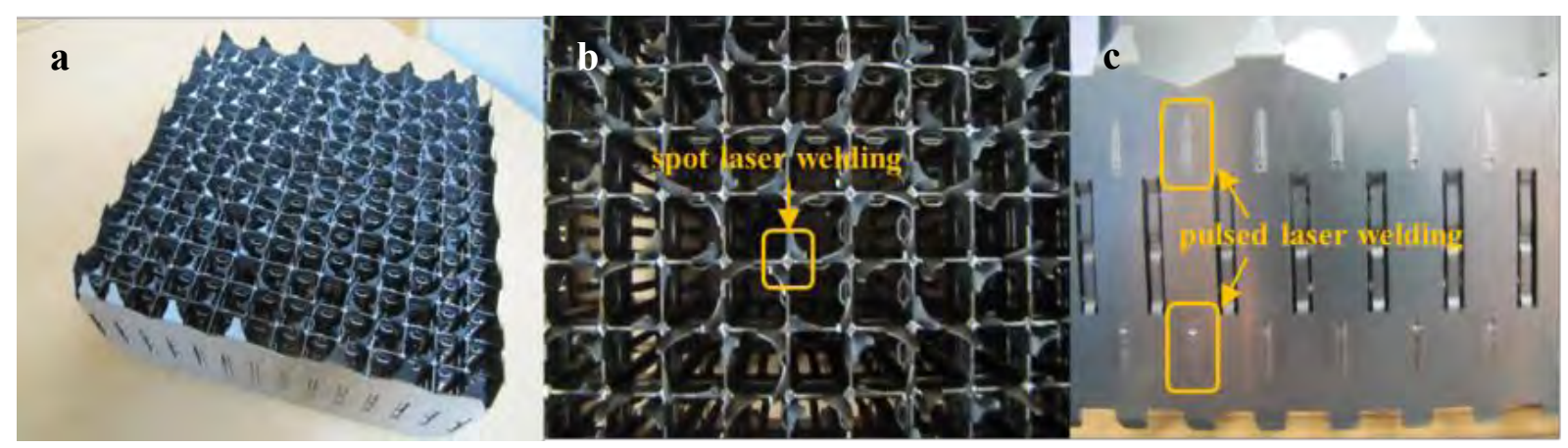

Figure 1: a) A 3-D view, b) top view, and c) side view of a $14 \times 14$ nuclear spacer grid.
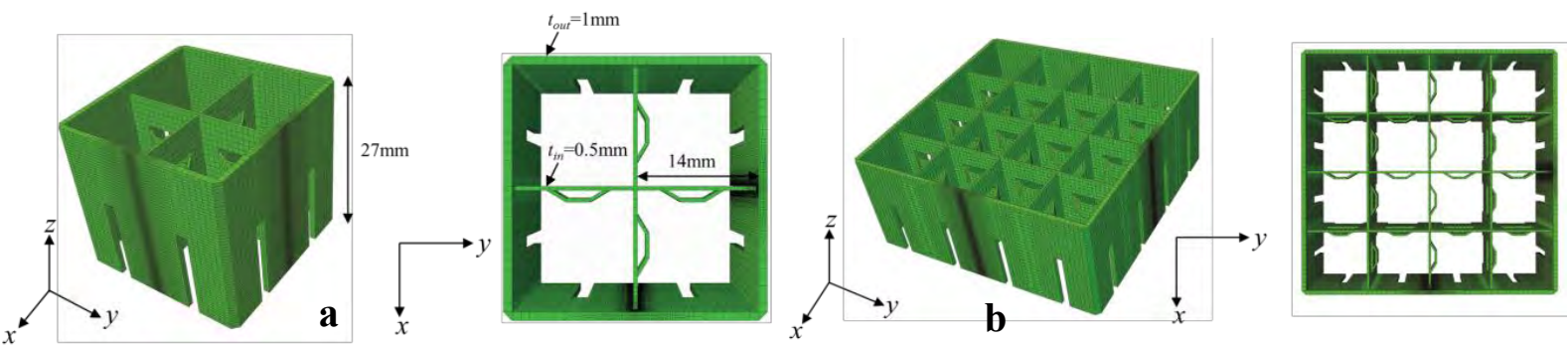

Figure 2: a) A reduced $2 \times 2$ model of a nuclear fuel spacer grid, b) a reduced $4 \times 4$ model of a nuclear fuel spacer grid.

\subsection{Multi-physics analysis of pulsed laser welding}

Using the model constructed above, simulations considering multiple mechanisms can be performed. The multi-physics simulations incorporate the latent heat of metallurgical transformations, the mechanical plasticity with temperature, and the metallurgical behaviour. For an accurate thermal and mechanical analysis, it is important to consider the thermomechanical properties with respect to the phase and temperature. Furthermore, nonlinear phenomena, including heat transfer, strain hardening with phase transitions, and plasticity, should be systematically calculated in robust multi-physics simulations. This enables the multi-physics simulation to provide the temperature field, phase distribution, and mechanical behaviours, such as welding distortions, residual stresses, and plastic strains, in the welded grid. Here, we perform multi-physics simulations to verify the efficiency and accuracy of the proposed computational technique. The numerical procedure of the multi-physics simulation is validated by comparison with the experiments conducted in our previous study.

In simulations of pulsed laser welding, an accurately defined heat source model must be used. Here, a modified conical Gaussian heat source model that properly depicts thermal energy penetration is employed. It is expressed as follows:

$$
Q=\frac{\eta P_{P}}{\pi r_{e}^{2} z_{e}} \exp \left(-\frac{r^{2}}{r_{e}^{2}}\right) \exp \left(-\frac{z}{z_{e}}\right)+Q_{1}
$$

where $r_{e}, z_{e}, \eta$, and $P_{p}$ refer to the initial radius, height of the heat source, mean absorption rate, and laser power, respectively. $Q_{1}$ is the modification factor defined by the shape of the welding spot observed from the experiment. The modified Gaussian heat source model can reflect the heat source density with regard to the characteristics of the laser and the 
exponential decay of thermal energy to the depth of the welding spot. Forty welding spots are simulated with a frequency $(f)$ of $5.0 \mathrm{~Hz}$, a duration $\left(T_{P}\right)$ of $5.0 \mathrm{~ms}$, and an interval between each pulse $\left(T_{F}\right)$ of $0.2 \mathrm{~s}$.

In order to efficiently develop a new simulation technique, pulsed laser welding simulations are performed with the $2 \times 2$ - and $4 \times 4$-unit spacer grid models. Calculating the pulsed laser welding procedure in a single seam line requires around 72 hours with an Intel i7$2600 \mathrm{~K}$ CPU with $3.40 \mathrm{GHz}$ and $12 \mathrm{~GB}$ of RAM. The thermal analysis providing the temperature field and phase distribution takes around 24 hours. The mechanical analysis providing the welding distortion and residual stress takes almost 48 hours. This inefficient computation severely restricts efforts to optimize the design of a welding procedure for minimizing the welding distortion.

The distortion of the grid induced by pulsed laser welding is investigated and the final welding distortion is determined when the temperature of the grid reaches a uniform room temperature $\left(20^{\circ} \mathrm{C}\right)$. In the simulation with the $2 \times 2$-unit spacer grid model, the difference between the maximum temperature, as shown in Fig. 3 a, and the room temperature becomes $0.01 \%$ after approximately 50 seconds, as shown in Fig. $3 \mathrm{~b}$, which indicates that the final distortion is achieved 50 seconds after pulsed laser welding. The temperature distributions of the $2 \times 2$ - and $4 \times 4$-unit models are shown in Fig. 4 . The pulsed laser welding procedure, including the forty welding spots, proceeded in the $z$-direction with one point constrained at the bottom centre of the welding surface.
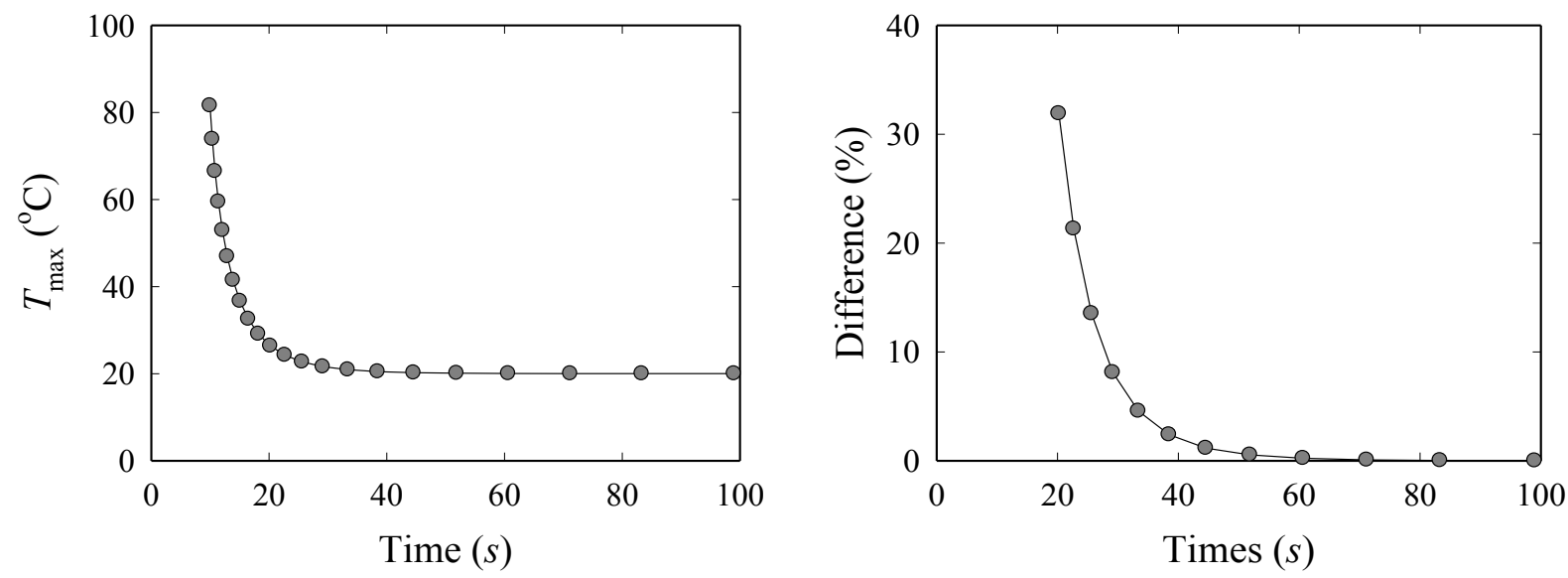

Figure 3: Maximum temperature of the $2 \times 2$-unit model (left), difference between the maximum temperature of a $2 \times 2$-unit model and room temperature (right).
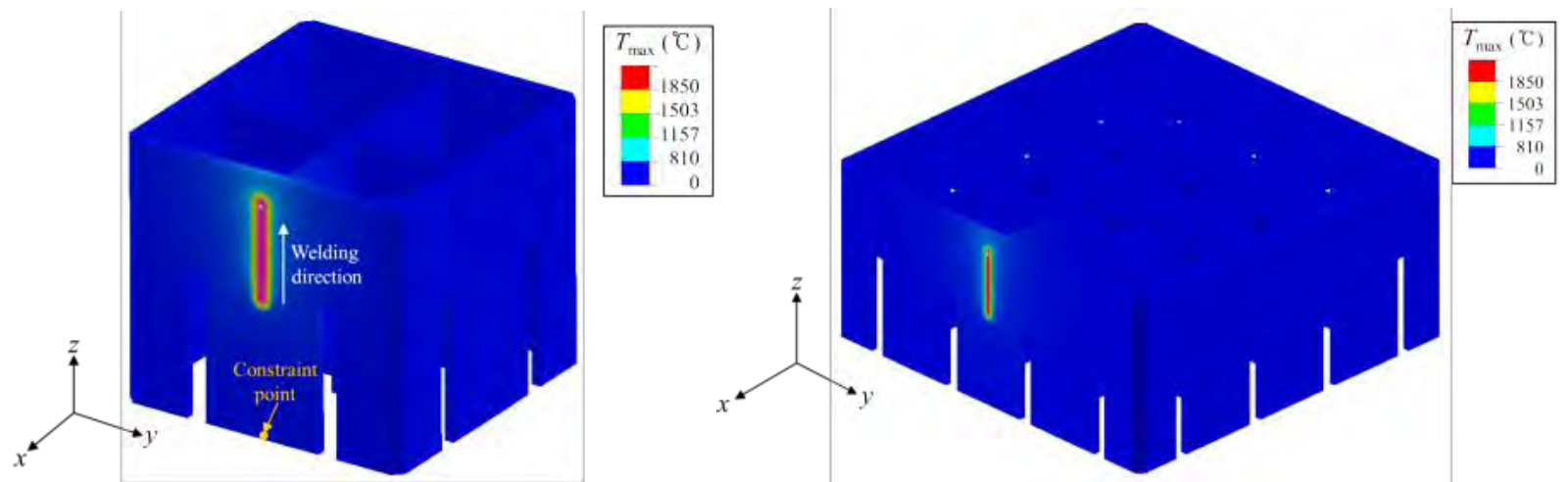

Figure 4: Temperature distributions in the $2 \times 2$-unit model (left) and $4 \times 4$-unit model (right).

Using the material properties of Zircaloy-4, we can find a HAZ and molten zone (MZ) where the temperature exceeds $810^{\circ} \mathrm{C}$ and $1850^{\circ} \mathrm{C}$, respectively. As shown in Fig. 4, the 
temperature distributions of both models are similar. The thermal energy introduced by the pulsed laser does not spread throughout the entire model but instead increases the local temperature near the welding spots. The width of a HAZ becomes $2.75 \mathrm{~mm}$, including the MZ, whose width is $0.8 \mathrm{~mm}$. Pulsed laser welding requires less thermal energy than continuous welding and induces a relatively small expansion of the HAZ region within the interval of each spot weld. Thus, the HAZ does not spread after the heat source is removed and the MZ and HAZ both maintain a uniform width.

Fig. 5 shows the welding distortions in the $x$-direction of the $2 \times 2$ - and $4 \times 4$-unit models. The deformation in the $y$-direction is relatively insignificant compared to that in the $x$ direction. An intense deformation is generated near the welding region. In the $2 \times 2$-unit model, both sides of the outer strip deform toward the welding region because of the condensation of the $\mathrm{MZ}$ and the contraction of the welding region with decreasing temperature after welding. In the $4 \times 4$-unit model, deformation occurs near the welding region in the outer strip as a result of the relatively unconstrained geometry. The deformation generated with this singleseam welding would accumulate during the sequential welding process.

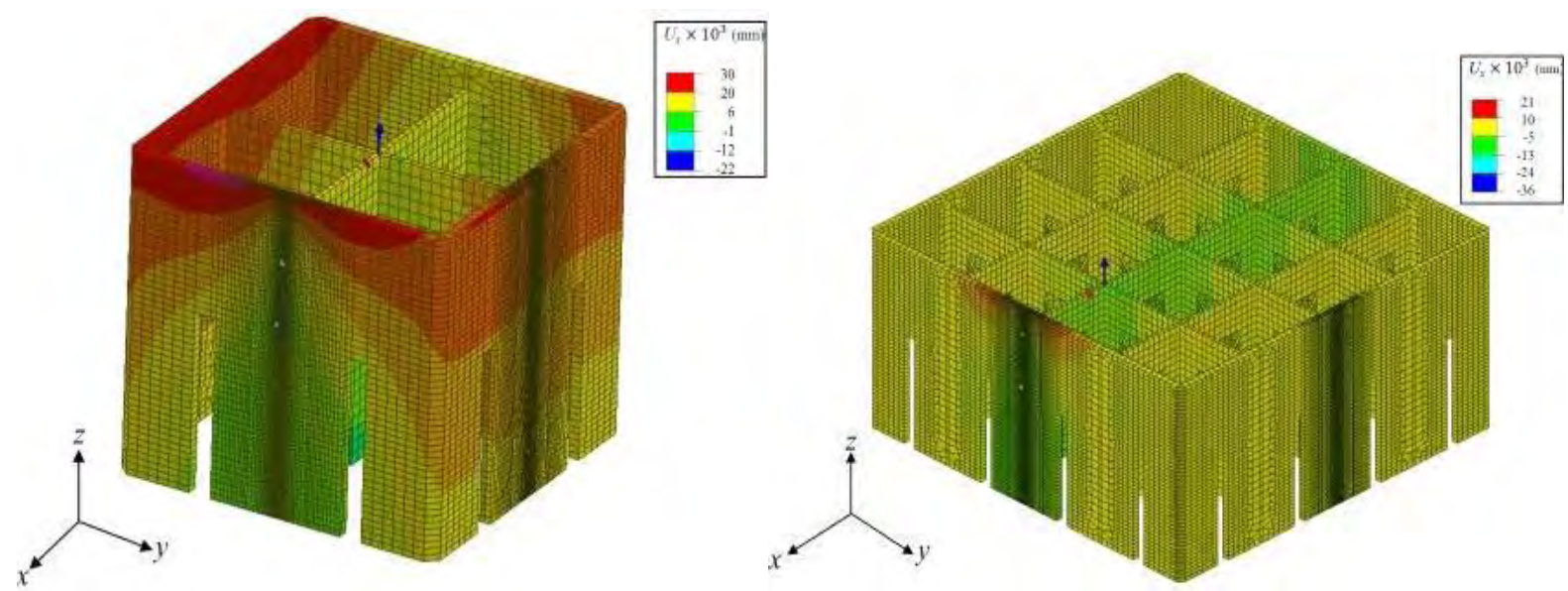

Figure 5: Deformations in the $x$-direction of the $2 \times 2$-unit model (left) and $4 \times 4$-unit model (right).

\subsection{Virtual welding distortion for the design of pulsed laser welding}

A multi-physics simulation validated by experimental data provides highly accurate information about welding distortion because it incorporates all the possible mechanisms involved, including the phase transition, convection, and radiation at each computational time step in addition to the mechanical and thermal calculations. However, it demands a highly sophisticated model with highly refined elements, resulting in impractical computational requirements for analysing an entire assembly procedure. To overcome the limitations of current multi-physics simulations, we propose a method that couples the multi-physics welding simulation and the mechanical distortion analysis with a virtual welding simulation. The mechanical deformation in pulsed laser welding is simulated with reasonable reliability and high efficiency by incorporating a virtual welding distortion derived from the multiphysics simulation, as shown in Fig. 6.

Fig. 7 shows a detailed coupling procedure for the multi-physics and mechanical distortion simulations. First, we define the control information derived from the multi-physics simulation and employ it in the mechanical distortion simulation. The control information is defined to cause the same distortion generated from the multi-physics simulation, which can be the deformation, stress, and strain of the welding region. The mechanical simulation is conducted with the control information applied to the boundary and initial conditions. From the mechanical simulation, the distortion is efficiently calculated and validated with the 
welding distortion obtained from the multi-physics simulation. The process is repeated until the control information that generates a reliable welding distortion is determined.

The von Mises stress distribution obtained from the multi-physics simulation for the control information is shown in Fig. 8 a. The stress is mainly generated at the welding region within $3 \mathrm{~mm}$ of the centreline of the welding. Thus, we consider the stress tensor of the welding surface elements within $3 \mathrm{~mm}$ of the centreline of the welding as the control information. The numbers of elements and nodes in this region are 22,014 and 26,489, respectively. We extract the twelve components of the stress $\left(\sigma_{x x}, \sigma_{y y}, \sigma_{z z}, \tau_{x y}, \tau_{y z}\right.$, and $\left.\tau_{z x}\right)$ and strain $\left(\varepsilon_{x x}, \varepsilon_{y y}, \varepsilon_{z z}, \gamma_{x y}, \gamma_{y z}\right.$, and $\gamma_{z x}$, ) tensors of each element and three components of the distortion vector $\left(u_{x}, u_{y}\right.$, and $\left.u_{z}\right)$. The extracted strain tensor and distortion vector are shown in Figs. $8 \mathrm{~b}$ and $8 \mathrm{c}$, respectively.

The virtual welding is simulated by a mechanical distortion calculation with the control information extracted from the multi-physics simulation. Simulations are performed with the $2 \times 2$ - and $4 \times 4$-unit models. The extracted stress and strain information is adopted as the initial conditions and the extracted distortion information is employed as the boundary conditions in the mechanical distortion analysis. The stress and strain information is applied to each element and the distortion information is applied to each node. From the employed information and constraints, we can perform the virtual welding simulation and reproduce the welding distortion without any consideration of multiple mechanisms, such as phase transition, convection, and radiation. The calculation for virtual welding distortion takes only around 30 minutes on an Intel i7-2600K CPU with $3.40 \mathrm{GHz}$ and $12 \mathrm{~GB}$ of RAM, including 20 minutes for a pre-processing stage that imports the control information for each node and element, leaving only 10 minutes for the calculation of mechanical behaviour.

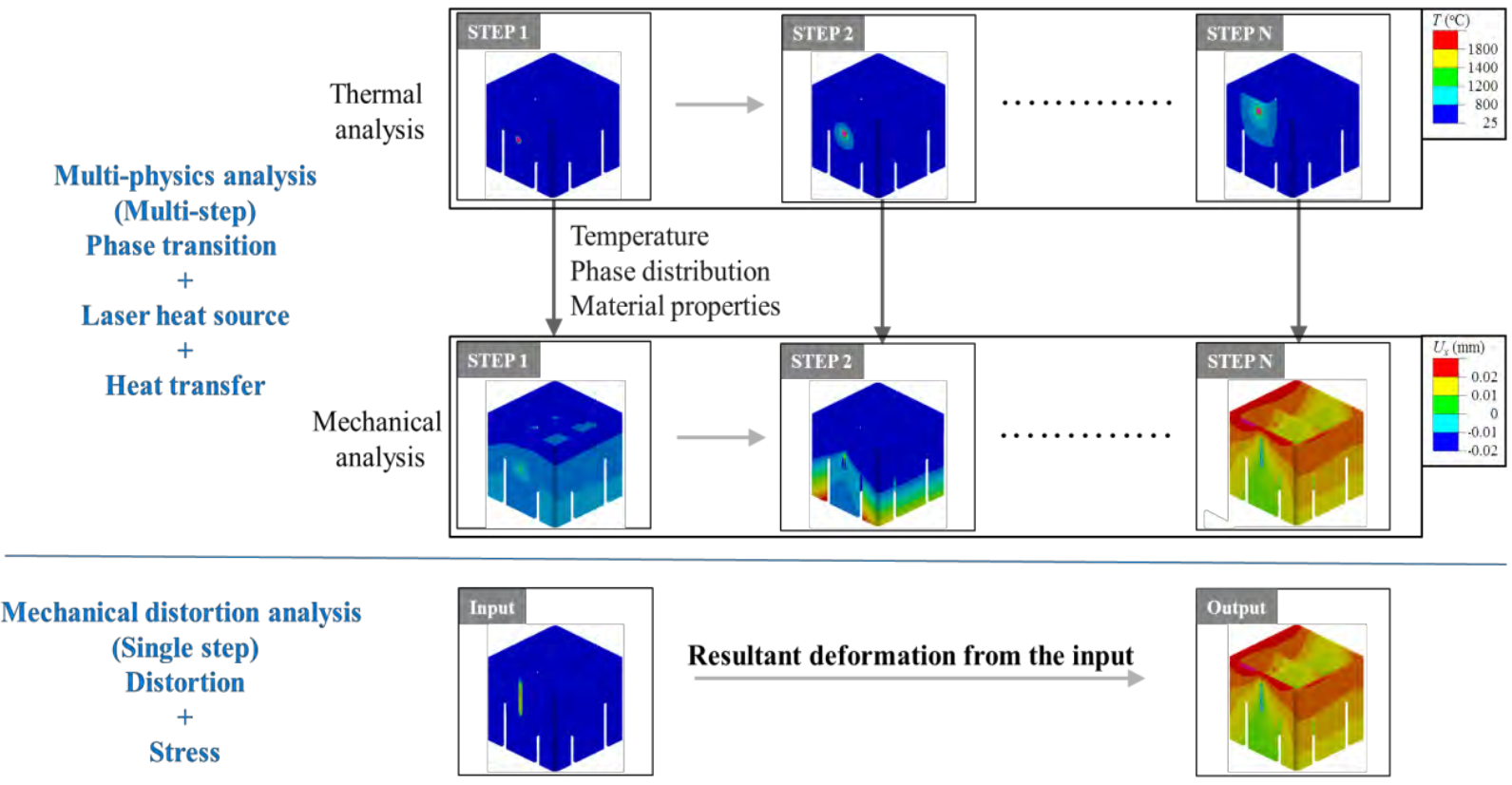

Figure 6: The one-step simulation technique for reproducing welding distortion. 


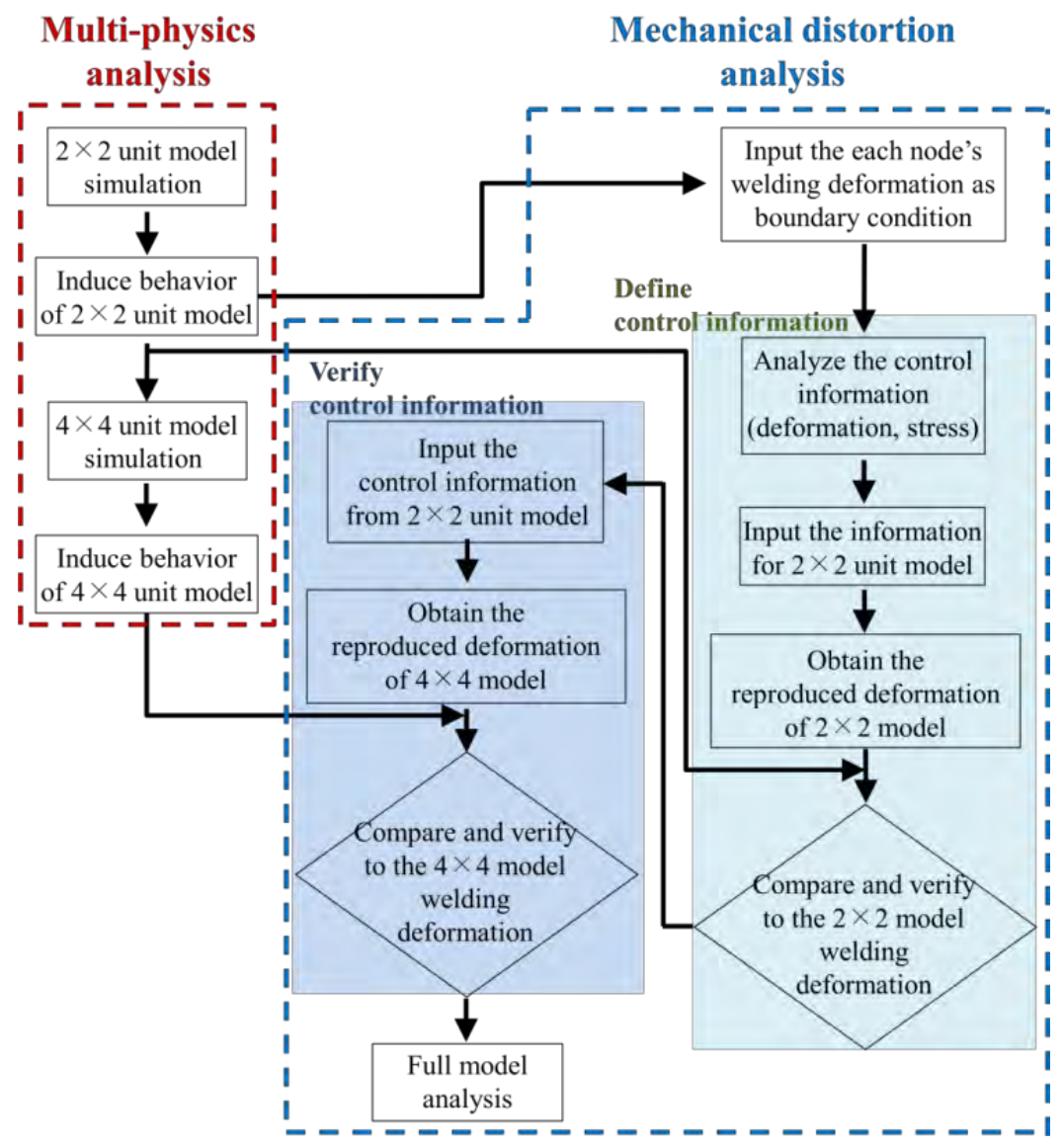

Figure 7: Procedure to define the virtual welding distortion with the multi-physics and mechanical distortion analysis.
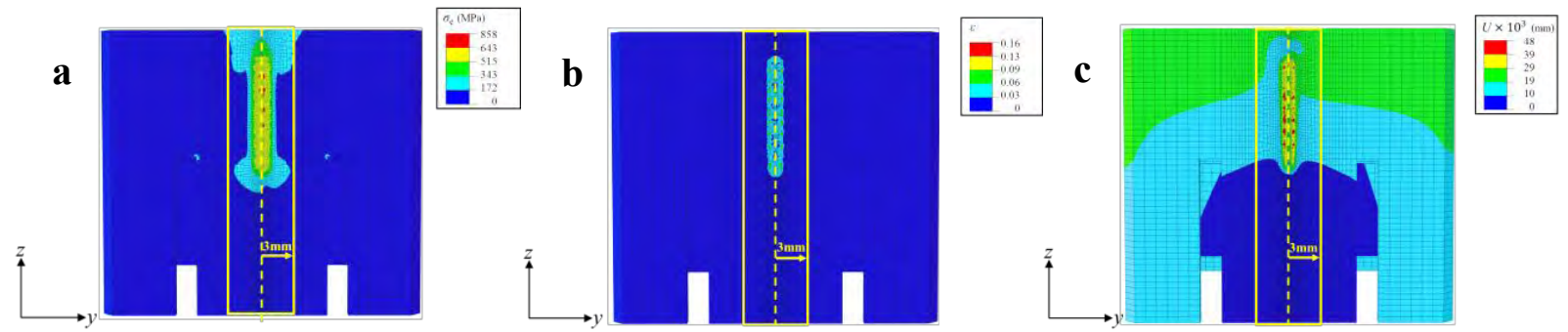

Figure 8: a) The stress field, b) the strain field and c) the welding distortion, calculated from the multiphysics simulation for control information.

\section{RESULTS AND DISCUSSION}

\subsection{Virtual welding distortion in the $2 \times 2$-unit model}

We can now apply the control information that reproduces the welding distortion to the mechanical distortion simulation and then compare the reproduced distortion to the welding distortion obtained from the multi-physics analysis. Because significant distortion is generated in the $x$-direction, the distortions in the $x-y$ plane are compared. Large distortions occur at the top corners of the opposite surface located furthest from the constraint point. When the $2 \times 2$-unit model is considered in a full $14 \times 14$-unit model, the distorted points of the $2 \times 2$-unit model are connected to other inner strips and induce a serious distortion in the entire model. Thus, we analyse the distortion at each corner point in the $x$-direction, $u_{x, 1}$ and $u_{x, 2}$, to quantify the distortion and compare it with the distortion obtained from the multi-physics analysis. 
The calculated welding distortions from the multi-physics simulation and the mechanical simulation are shown in Fig. 9. The distortion calculated from the mechanical simulation with the control information is compared to that from the multi-physics simulation in Fig. 10. When the stress or strain field is used for the control information, the maximum distortion in the opposite surface is similar to that from the multi-physics simulation. However, distortions in other regions exhibit considerable differences from those of the multi-physics simulation; these are approximately $10 \%$ for distortions in each corner, $u_{x, 1}$ and $u_{x, 2}$, and 23-36\% for each side of the inner strip. However, when the welding distortion in the welding region is employed as the control information in the multi-physics simulation, the reproduced distortion in the opposite surface and even in the inner strip shows a great similarity to the distortion calculated from the multi-physics simulation. The differences in the distortions at each corner, $u_{x, 1}$ and $u_{x, 2}$, are only $0.01 \%$ and $1.03 \%$, respectively.

When we impose the stress field at the welding region, the relaxing stress spreads to the entire model. From the relaxation, the intensive stress decays and the positions of the nodes used in the multi-physics simulation are also altered. Thus, the calculation using the stress or strain fields as the control information gives rise to unreliable distortions. The difference in the calculated distortion of the reproduced unit model can cause critical errors when the model is expanded to an entire assembly because the error is proportional to the distance from the pulsed welding region. When the welding distortion is employed as the control information for the multi-physics simulation, the positions of each node are adopted, as determined from the multi-physics analysis, and the reproduced distortion displays a high accuracy.
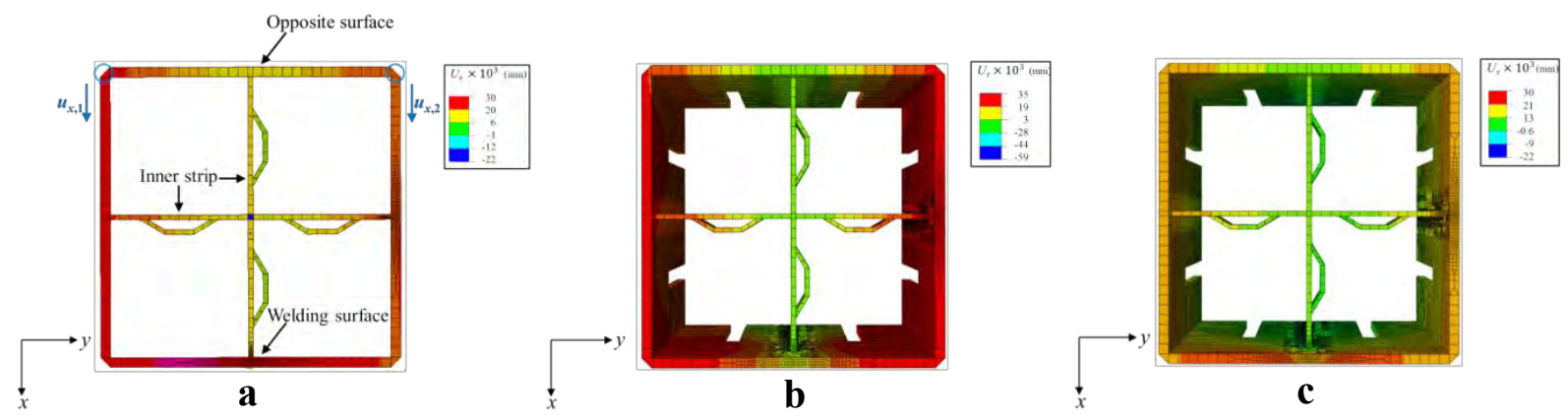

Figure 9: Distortion of the $2 \times 2$-unit model in the $x$-direction from a) multi-physics analysis and calculated with b) initial stress and strain, and c) the distortion of the welding spot.
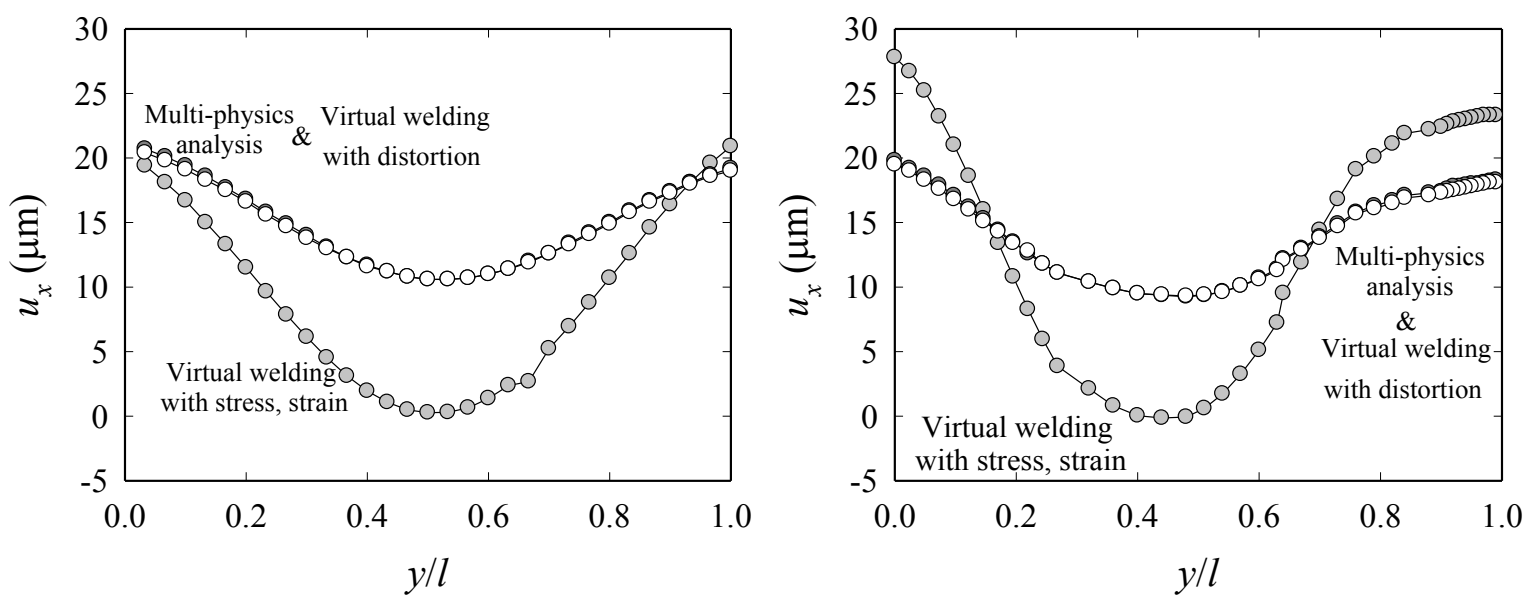

Figure 10: Distortions of the opposite surface (left) and the inner strip in the $x$-direction (right). 


\subsection{Virtual welding distortion in the $4 \times 4$-unit model}

To validate the control information, we applied the control information obtained from the $2 \times 2$-unit model to the $4 \times 4$-unit model. The acquired information on the stress, strain, and distortion is employed within a unit grid distance. Thus, the information is independent of the number of the grid, the welding site, and the sequence. The welding region of the $4 \times 4$-unit model is composed of the same numbers of elements and nodes as the $2 \times 2$-unit model, so the control information from the $2 \times 2$-unit model can be applied. In addition, each welding region can be indexed to apply the control information at each corresponding pulsed welding region. The reproduced distortions in the $x$-direction of the $4 \times 4$-unit model with different types of control information are presented in Fig. 11, and the distortions of the opposite surface and the middle of the inner strip along the $y$-axis are analysed and verified in Fig. 12.

When the stress or strain information is adopted as the control information, the differences in $u_{x, 1}$ and $u_{x, 2}$ from the multi-physics simulation become $7-44 \%$. When the distortion is employed as the control information, the difference becomes less than $6 \%$. As the model is expanded, the error increases but the distortion still provides reliable simulation results, whereas the tendency of the reproduced distortion varies when the stress or strain field is employed as the control information. When we adopt the distortion of the welding region as the control information, the reproduced distortion does not show any considerable differences from the welding distortion calculated from the multi-physics simulation.

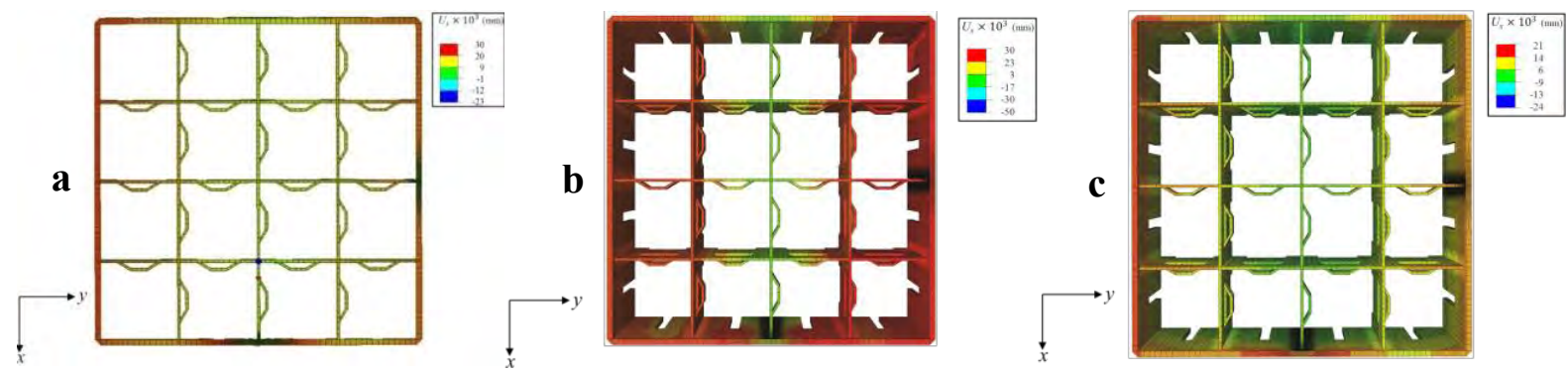

Figure 11: Distortion of the $4 \times 4$-unit model in the $x$-direction from a) the multi-physics analysis and calculated with b) the initial stress and strain, and c) the distortion of the welding spot.
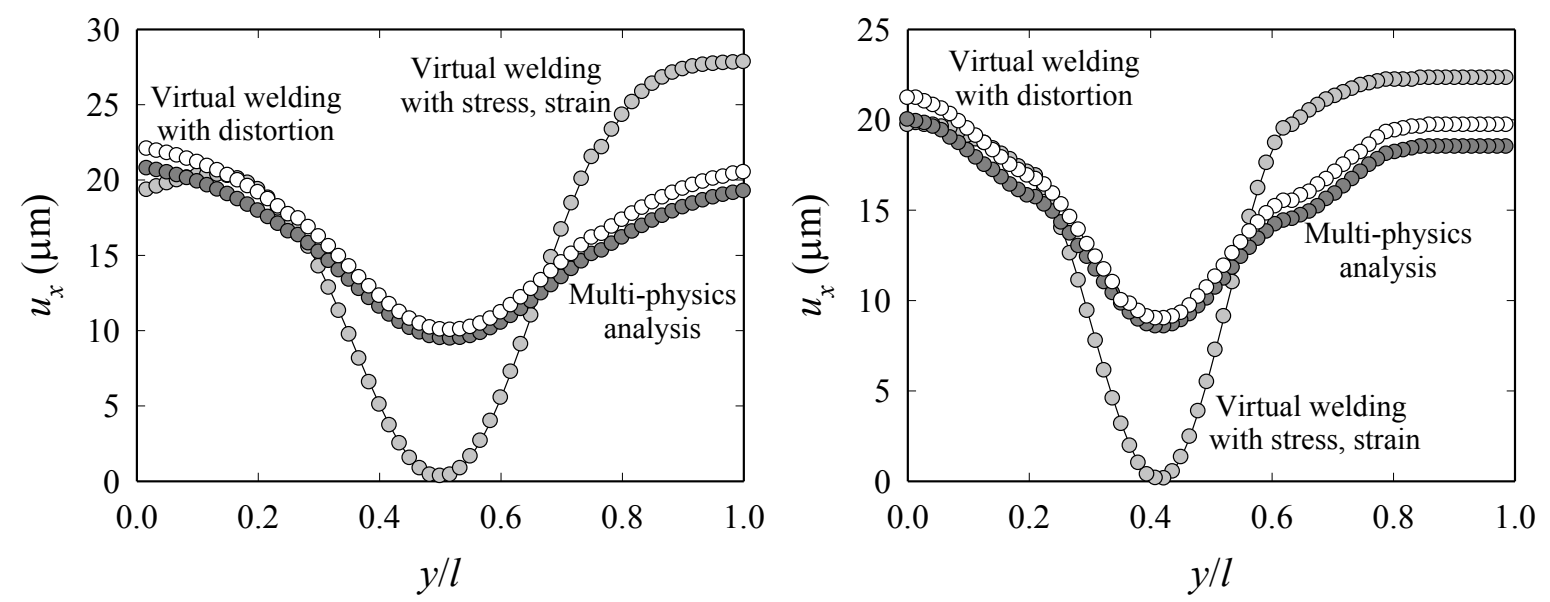

Figure 12: Distortion of the opposite surface (left) and the middle of inner strip in the $x$-direction (right).

However, when we adopt the stress or strain field to reproduce the distortion, the error is increased significantly at the surface of each side. The distortion reproduced using the stress or strain field becomes almost zero around the middle of the inner and outer strips, regardless of the size of the model. If the dimensions of the grid are increased, then the error of the reproduced distortion considerably increases because the applied stress or strain does not 
restore mechanical behaviour due to the relaxation. As the model size is increased further, the error will be significant.

Thus, the distortion reproduced using the stress or strain field is inappropriate for reproducing the welding distortion, whereas the distortion reproduced using the control information from the distortion in the welding region in the multi-physics simulation provides sufficient reliability for efficient welding simulation. Therefore, in this paper, the control information from the distortion in the welding region calculated from the multi-physics simulation is defined as the virtual welding distortion. In the next section, we apply this to the welding simulation of a complete model of the entire nuclear spacer grid.

\subsection{Welding simulation for a complete model of the nuclear fuel spacer grid}

To investigate the welding deformation of the nuclear fuel spacer grid, we constructed a complete model, as shown in Fig. 13 a. We employed a $19 \times 19$ spacer grid, which is larger than an ordinary $14 \times 14$ grid, to emphasize the efficiency of the developed method. The model consists of 2,135,489 elements and 2,775,316 nodes. The full model contains eighteen pulsed welding regions on each surface, with each region having the same element structures as the $2 \times 2$-unit model. However, the laser welding procedure for the complete model cannot be efficiently calculated by a multi-physics simulation, even with significant computational resources. Thus, we employed the virtual welding distortion from the $2 \times 2$-unit model and directly performed a mechanical simulation of the complete model. The mechanical analysis was performed with a personal computer with an Intel i7-2600K CPU with $3.40 \mathrm{GHz}$ and 12 GB of RAM.

The welding simulation for one pulsed seam welding took 18 hours to provide the welding distortion over the entire model, as shown in Fig. $13 \mathrm{~b}$. The distortion in the $x$-direction and the magnification $(\times 100)$ are proportional to the distance in the $y$-direction from the welding region. The distortion tendency of the grid is different at the left and right sides of the welding region because the distances to the outer strips differ. The asymmetric geometry of the grid also causes the asymmetric distortion. The distortion in the $x$-direction is approximately 0.1 $\mathrm{mm}$ at the centre area of the grid. There are 72 regions of pulsed laser welding. Thus, by employing the virtual welding distortion method, we can perform a sequence of simulations of the laser welding assembly procedure and investigate the final accumulated welding distortion in the nuclear fuel spacer grid.
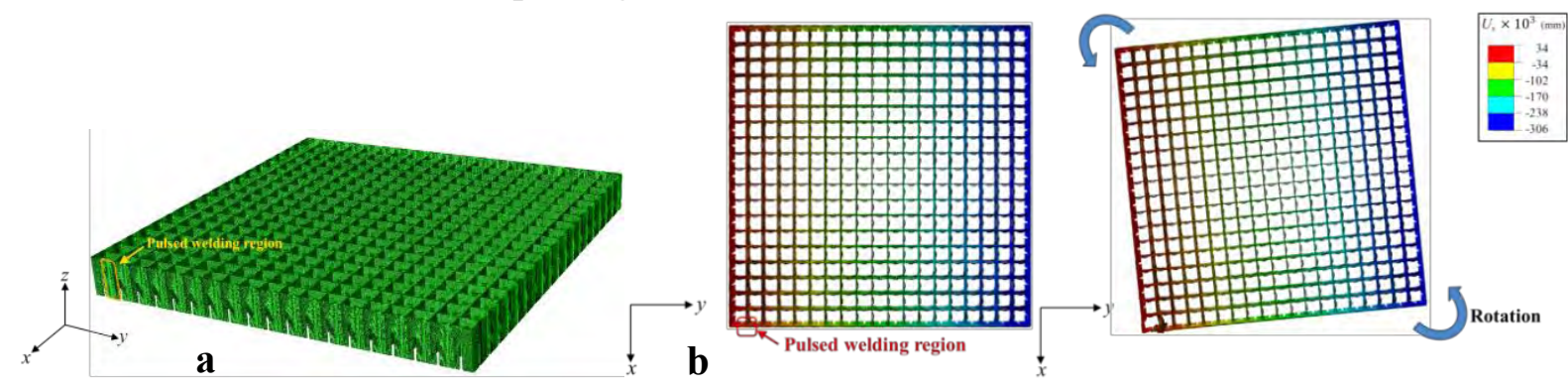

Figure 13: a) The complete model of an $18 \times 18$ nuclear fuel spacer grid, b) distortion of the $18 \times 18$ model in the $x$-direction and the magnification $(\times 100)$ from the virtual welding analysis.

\section{CONCLUSIONS}

In this study, the following conclusions were made:

- The multi-physics simulation for laser welding can be applied only in very limited cases, even though it provides high accuracy comparable to experimental data. For example, a simulation of seam welding with a single pulsed laser on a reduced $2 \times 2$ model of a nuclear fuel spacer grid with dimensions of $30 \mathrm{~mm} \times 30 \mathrm{~mm} \times 27 \mathrm{~mm}$ requires 24 hours to 
calculate the temperature distribution and an additional 48 hours for the welding distortion and residual stress. It is therefore clear that the multi-physics simulation demands unfeasibly large computational resources. Thus, an alternative simulation technique is required to investigate the welding distortion of the nuclear fuel spacer grid with reasonable efficiency and reliability.

- An efficient welding simulation was proposed in this paper, and is based on coupling the multi-physics simulation with a mechanical simulation providing the control information. For the control information, a virtual welding distortion in the welding region was developed from an investigation of the accuracy of other types of control information, such as the stress and strain fields. The virtual welding distortion was determined from a multiphysics simulation conducted on a $2 \times 2$-unit model. The virtual welding distortion provided a reliable representation of pulsed laser welding in the welding region and enabled efficient mechanical simulation, producing the welding distortion in the assembly with high accuracy.

- The developed simulation procedure required only 30 minutes to calculate the welding distortion of the $2 \times 2$ - and $4 \times 4$-unit models, while the multi-physics simulation took over 72 hours. The differences between the calculated welding distortions in the model are only within $1 \%$ and $6 \%$ for the $2 \times 2$ - and $4 \times 4$-unit models, respectively.

- As the virtual welding distortion was determined within the welding region of a single unit, it could be extended from the $2 \times 2$-unit model to the complete model of the nuclear fuel spacer grid. The welding distortion of the $4 \times 4$-unit model, which was calculated with the virtual welding distortion derived from the $2 \times 2$-unit model, showed only less than $6 \%$ error compared to the welding distortion from the multi-physics simulation.

- The developed simulation procedure with the virtual welding distortion was successfully applied to calculate the welding distortion of the complete model of the nuclear fuel spacer grid. The calculation of the pulsed laser seam welding took only 18 hours. The proposed welding simulation procedure can be applied to the analysis of a laser welding sequence, and can provide an efficient and reliable computational analysis and optimization of the laser assembly procedure of a nuclear fuel spacer grid.

\section{ACKNOWLEDGEMENTS}

This research was supported by the Basic Science Research Program and the National Nuclear R\&D Program through the National Research Foundation (NRF) of Korea, funded by the Ministry of Education, Science and Technology (No. 2013R1A1A2011263 and No. 2014R1A2A2A09052374), and by a research grant (No. 2009-0083510) funded by the Korean government (MEST) through the Multi-phenomena CFD Engineering Research Center.

\section{REFERENCES}

[1] Ghosh, A.; Hloch, S. (2013). Prediction and optimization of yield parameters for submerged arc welding process, Technical Gazette, Vol. 20, No. 2, 213-216

[2] Bukvic, A.; Burzic, Z.; Prokic-Cvetkovic, R.; Popovic, O.; Burzic, M.; Jovicic, R. (2012). Welding technology selection effect on fracture-toughness parameters of bi-material welded joints, Technical Gazette, Vol. 19, No. 1, 167-174

[3] Weckman, D. C.; Kerr, H. W.; Liu, J. T. (1997). The effects of process variables on pulsed Nd:YAG laser spot welds: Part II. AA 1100 aluminum and comparison to AISI 409 stainless steel, Metallurgical and Materials Transactions B-Process Metallurgy and Materials Processing Science, Vol. 28, No. 4, 687-700, doi:10.1007/s11663-997-0043-1

[4] Tzeng, Y.-F. (2000). Process characterisation of pulsed Nd:YAG laser seam welding, International Journal of Advanced Manufacturing Technology, Vol. 16, No. 1, 10-18, doi:10.1007/PL00013126 
[5] Fuerschbach, P. W.; Eisler, G. R. (2002). Effect of laser spot weld energy and duration on melting and absorption, Science and Technology of Welding and Joining, Vol. 7, No. 4, 241-246, doi:10.1179/136217102225004293

[6] Tadamalle, A. P.; Reddy, Y. P.; Ramjee, E. (2013). Influence of laser welding process parameters on weld pool geometry and duty cycle, Advances in Production Engineering \& Management, Vol. 8, No. 1, 52-60, doi:10.14743/apem2013.1.153

[7] Vegelj, D.; Zajec, B.; Gregorcic, P.; Mozina, J. (2014). Adaptive pulsed-laser welding of electrical laminations, Strojniski Vestnik - Journal of Mechanical Engineering, Vol. 60, No. 2, 106-114, doi:10.5545/sv-jme.2013.1407

[8] Rosenthal, D.; Cambridge, M. (1946). The theory of moving sources of heat and its application to metal treatments, Transactions of the ASME, Vol. 68, No. 11, 849-866

[9] Mazumder, J.; Steen, W. M. (1980). Heat transfer model for cw laser material processing, Journal of Applied Physics, Vol. 51, No. 2, 941-947, doi:10.1063/1.327672

[10] Frewin, M. R.; Scott, D. A. (1999). Finite element model of pulsed laser welding, Welding Journal, Vol. 78, No. 1, 15s-22s

[11] He, X.; Fuerschbach, P. W.; DebRoy, T. (2003). Heat transfer and fluid flow during laser spot welding of 304 stainless steel, Journal of Physics D: Applied Physics, Vol. 36, No. 12, 1388-1398, doi: $10.1088 / 0022-3727 / 36 / 12 / 306$

[12] Ehlen, G.; Ludwig, A.; Sahm, P. R. (2003). Simulation of time-dependent pool shape during laser spot welding: Transient effects, Metallurgical and Materials Transactions A-Physical Metallurgy and Materials Science, Vol. 34, No. 12, 2947-2961, doi:10.1007/s11661-003-0194-x

[13] Moraitis, G. A.; Labeas, G. N. (2008). Residual stress and distortion calculation of laser beam welding for aluminum lap joints, Journal of Materials Processing Technology, Vol. 198, No. 1-3, 260-269, doi:10.1016/j.jmatprotec.2007.07.013

[14] Han, Q.; Kim, D.; Kim, D.; Lee, H.; Kim, N. (2012). Laser pulsed welding in thin sheets of Zircaloy-4, Journal of Materials Processing Technology, Vol. 212, No. 5, 1116-1122, doi:10.1016/j.jmatprotec.2011.12.022

[15] Ferro, P.; Tiziani, A. (2012). Metallurgical and mechanical characterization of electron beam welded DP600 steel joints, Journal of Materials Science, Vol. 47, No. 1, 199-207, doi:10.1007/s10853-011-5787-y

[16] Joshi, S.; Hildebrand, J.; Aloraier, A. S.; Rabczuk, T. (2013). Characterization of material properties and heat source parameters in welding simulation of two overlapping beads on a substrate plate, Computational Materials Science, Vol. 69, 559-565, doi:10.1016/j.commatsci. 2012.11.029

[17] Siefken, L. J.; Coryell, E. W.; Harvego, E. A.; Hohorst, J. K. (2001). SCDAP/RELAP5/MOD 3.3 Code Manual: MATPRO - A Library of Materials Properties for Light-Water-Reactor Accident Analysis, U.S. Nuclear Regulatory Commission Office, Washington, DC 